\title{
Myocardial hypertrophy, fibrosis and infarction following exposure of the heart to radiation for Hodgkin's disease
}

\author{
Luke O’Donnell ${ }^{1}$, Terence O’Neill ${ }^{1}$, Mary Toner $^{2}$, Sean O'Briain ${ }^{2}$ and Ian Graham ${ }^{1}$ \\ ${ }^{1}$ Adelaide Hospital, ${ }^{2}$ St. James's Hospital and the Departments of Medicine and Histopathology, Trinity College, \\ Dublin, Republic of Ireland
}

\begin{abstract}
Summary: A 35 year old man was treated for stage IIA Hodgkin's disease by radiation to the upper thorax, axillae and neck. Three years later he presented with intractable and ultimately fatal congestive heart failure.

Autopsy revealed massive biventricular hypertrophy with widespread subendocardial fibrosis and myocardial infarction, but with little coronary artery disease. Such a complex of features has not previously been described after radiation therapy and cannot be adequately explained by other known causes of heart muscle disease. Ventricular hypertrophy with extensive subendocardial fibrosis may be part of the spectrum of radiation heart disease.
\end{abstract}

\section{Introduction}

Radiation-induced heart disease is well described and usually manifests as acute or chronic pericarditis (Cohn et al., 1967; Stewart \& Fajardo, 1971; Applefeld et al., 1981) although myocarditis (Burch et al., 1968), myocardial fibrosis (Rubin et al., 1963), valvulitis (Brosius et al., 1981), coronary artery disease (Tracy et al., 1974) and myocardial infarction (Dollinger et al., 1966; McReynolds et al., 1976) also occur.

Echocardiographic and radionuclide ventriculography abnormalities have also been reported in a substantial proportion of asymptomatic patients who previously received radiation therapy (Burns et al., 1983; Gottdiener et al., 1983). We report a case of cardiomyopathy with widespread subendocardial fibrosis, massive biventricular hypertrophy, mural thrombi and myocardial infarction, a complex of features not previously described following radiation therapy.

\section{Case report}

A 35 year old previously healthy male presented with a 10 year history of axillary swelling. He had no cardiac symptoms and cardiovascular examination was normal. There was no history of hypertension. He had smoked 40 cigarettes per day for 20 years and

Correspondence: I. Graham, F.R.C.P.I., F.C.C.P., Department of Cardiology, Adelaide Hospital, Dublin 8, Republic of Ireland.

Accepted: 30 May 1986 consumed $40 \mathrm{~g}$ of alcohol per day for 15 years. Assessment, including laparotomy and splenectomy, revealed right axillary and supra-clavicular lymphadenopathy due to lymphocytic predominant Stage IIA Hodgkin's disease. Radiation treatment was given as 3,500 rads in divided doses in a 'mantle' distribution to the neck and upper thorax which did not exclude the heart. Both axillae were exposed to 3,500 rads and a boost of 600 rads to the right axilla was given. $\mathrm{He}$ received no cytotoxic therapy.

He remained well until three and a half years later when he developed dyspnoea on exertion, paroxysmal nocturnal dyspnoea and chest tightness. The pulse was regular with a rate of 120 beats/min, blood pressure was $90 / 60 \mathrm{mmHg}$, and the jugular venous pressure was raised to $10 \mathrm{~cm}$. The apex beat was displaced laterally and was dyskinetic with a prominent gallop-rhythm. Bibasal crepitations were heard in the lung fields and ankle oedema was noted.

Chest X-ray showed gross cardiomegaly with pulmonary congestion. Left bundle branch block and biatrial overload were present on the electrocardiogram. Echocardiography and radionuclide ventriculography showed a severely dilated left ventricle with uniformly poor function. Routine haematological and biochemical investigations were normal as were serum iron, total iron binding capacity, serum thyroxine, Coxsackie, cytomegalovirus, $Q$ fever and toxoplasma titres, and rectal biopsy for amyloid.

Conventional therapy with digoxin and diuretics was instituted. Over the following year, he was admitted to hospital on eight occasions with progres- 
sive right and left heart failure requiring vasodilator therapy and eventually inotropic support. During this period he developed pulmonary Mycobacterium xenopi infection which responded well to isoniazid and ethambutol. Rifampicin was discontinued because of the development of jaundice. Cardiac transplantation was considered and declined. One year after presentation to us the patient died from unresponsive cardiac failure complicated by a chest infection.

Post-mortem examination showed no evidence of Hodgkin's disease, mycobacterial infection or liver disease. The heart weighed $820 \mathrm{~g}$. There was biventricular hypertrophy with a muscle thickness of $1.9 \mathrm{~cm}$ (left ventricle), $0.6 \mathrm{~cm}$ (right ventricle) and four chamber dilatation. Grossly, the ventricular walls showed two fibrotic areas: a transmural area of the posterior wall $(7 \times 4 \mathrm{~cm})$ extending to the apex and a subendocardial area in the anterior half of the septum $(6 \times 5 \mathrm{~cm})$. Both areas were covered by mural throm-

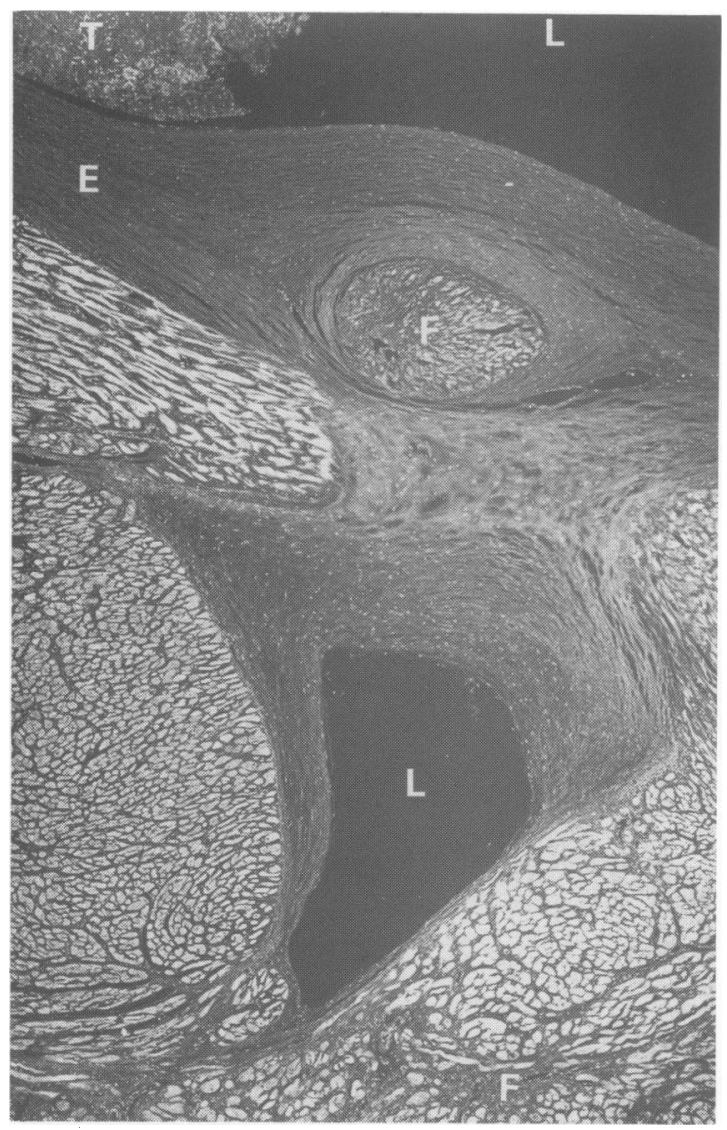

Figure 1 Section of left ventricle. $L$ indicates left ventricular lumen. Mural thrombus (T) overlies thickened endocardium (E). Focal interstitial fibrosis (F) is seen within the myocardium. Haematoxylin and eosin $\times 50$. bus. The coronary arteries showed only mild focal atherosclerosis with a maximum stenosis of $50 \%$ in the left anterior descending artery $6 \mathrm{~cm}$ from its origin and $30 \%$ stenoses of the circumflex and right coronary arteries $2 \mathrm{~cm}$ from the origin of each. There was a mild obliterative fibrous pericarditis. The valves were normal.

Ventricular wall histology showed striking subendocardial fibrosis varying from 1 to $10 \mathrm{~mm}$ in thickness involving all of the left ventricle apart from the lateral wall and involving most of the right ventricle. There was widespread entrapment of myocardial trabeculae by the fibrosis. This pattern of fibrosis with myocardial trabecular entrapment reached a thickness of $10 \mathrm{~mm}$ in the anterior half of the interventricular septum giving the gross appearance of a discrete area of infarction (Figure 1). The posterior wall showed transmural fibrosis with loss of myocardium consistent with an area of ischaemic infarction. Special stains indicated that the subendocardial fibrosis was composed mainly of collagen; however, moderate amounts of elastic fibres were present close to the underlying muscle. Scattered foci of lymphocytes and plasma cells were present in association with the areas of subendocardial fibrosis. The myocardial cells showed enlarged pleomorphic nuclei consistent with hypertrophy. There were occasional small foci of fibrosis scattered throughout the myocardium. The atria demonstrate mild muscle hypertrophy but the atrial endocardiun appeared normal.

Histology of the coronary arteries showed eccentrie atheromatous plaque formation in the areas seen grossly. There was a mild degree of intimal thickening of the small vessels throughout the myocardium with a modest $(10 \%)$ reduction in lumen size.

\section{Discussion}

The most striking features in our patient were the presence of widespread subendocardial fibrosis in a massively hypertrophied and dilated heart, a complex which has not been described previously in association with radiation. This was associated with mural thrombus and an area of transmural infarction despite mild coronary artery disease. Fibrous pericarditis has frequently been described in patients who have received radiation (Cohn et al., 1967; Applefeld et al., 1981) but was only of a mild degree in our patient.

The area of myocardial infarction described is not easily explained as there was no discrete clinical episode to suggest myocardial infarction and only mild focal coronary artery disease was noted. However, an embolus from the left ventricular mural thrombus, a completely resolved coronary artery thrombosis in a heavy smoker or direct radiation damage are all possibilities. The degree of both 
hypertrophy and subendocardial fibrosis is far in excess of secondary changes which might be expected to occur if a myocardial infarction was the primary event.

Myocardial hypertrophy with dilatation, subendocardial fibrosis and mural thrombus may be seen in dilated cardiomyopathy and in the late stages of viral myocarditis. Typical or atypical reaction to viral or other infections might be expected in a patient with Hodgkin's disease, even in remission, as evidenced by the occurrence of pulmonary infection due to an atypical mycobacterium in this case. Therefore, coincidental or opportunistic viral myocarditis cannot be excluded. However, serology for several suspect viruses was negative, inflammatory infiltrate was minimal and the degree of subendocardial fibrosis was greater than usually described in association with viral myocarditis or dilated cardiomyopathy. The gradual clinical onset would also be unusual for viral myocarditis.

There was no clinical or pathological evidence of amyloidosis, hypertrophic obstructive cardiomyopathy, hypertension or valvular disease. Alcohol is unlikely to have had a primary role in the aetiology in view of the quantity ingested and the fact that this degree of hypertrophy and subendocardial fibrosis are unusual in myocardial disease due to alcohol abuse. However, a secondary role for alcohol in accelerating the heart failure is possible. Malnutritional effects often associated with alcohol intake are unlikely as nutrition was well maintained in our patient. Neither clinical presentation nor autopsy findings suggest carcinoid syndrome or Loffler's endocarditis.

Many factors suggest the possibility of a direct radiation reaction in this case. There is a history of exposure to significant doses of radiation without protection of the heart. The onset of the illness three and a half years after radiation is consistent with the time lag of delayed onset radiation-induced disease. In addition there is no evidence indicative of viral myocarditis or other known forms of cardiomyopathy.

Heart muscle disease other than fibrosis has rarely been described as a complication of radiation therapy. Rubin et al. (1963) reported the post-mortem findings of three patients exposed to mediastinal radiation, one of whom died from a cardiac cause (progressive

\section{References}

APPLEFELD, M.M., COLE, J.F., POLLOCK, S.H., SUTTON, F.J., SLAWSON, R.G., SINGLETON, R.T. \& WIERNIK, P.H. (1981). The late appearance of chronic pericardial disease in patients treated by radiotherapy for Hodgkin's disease. Annals of Internal Medicine, 94, 338.

APPLEFELD, M.M., SLAWSON, R.G., SPICER, K.M., SIN- conduction defect associated with conducting tissue fibrosis). The predominant finding in these patients was widespread patchy fibrosis of the myocardium. The hearts were only mildly enlarged $(295 \mathrm{~g}-375 \mathrm{~g})$. Burch et al. (1968) described the electron microscopy findings in a patient who died from cardiac failure 5 years after mediastinal radiation for thyroid cancer but no reference to gross enlargement of the heart or subendocardial fibrosis was made. In a series of 25 patients followed for a median period of 96 months after mediastinal radiation for Hodgkin's disease Applefeld et al. (1982) noted that one patient had clinical evidence of cardiomyopathy. No histological information was available as the patient was still alive.

There appear to be many possible pathological responses of the heart to radiation, including pericarditis (Cohn et al., 1967; Applefeld et al., 1981), diffuse myocardial fibrosis (Rubin et al., 1963) and coronary artery disease (Tracy et al., 1974). Why any one finding should predominate in a particular patient is unknown, and it is uncertain why our patient had predominant subendocardial fibrosis with myocardial hypertrophy. Alcohol may have rendered the heart more susceptible to the described injury. Tissue reaction to radiation is always more pronounced in previously inflamed or damaged areas. The endocardium as a modified endothelium may also be subject to damage similar to the endothelial damage frequently found as a radiation effect (Cohn et al., 1967). The massive hypertrophy may have been secondary to a non-compliant left ventricular wall stiffened by the subendocardial fibrosis.

Treated Hodgkin's disease is nowadays associated with an excellent prognosis. Many of the long-term problems in these patients now result from complications of therapy rather than of the primary disease itself (Carmel \& Kaplan, 1976). As more of these cured patients accumulate it is important to define carefully and be aware of the range of therapy-induced complications for two reasons. Firstly, so that the therapy for the initial disease can be modified and secondly so that complications of therapy will be recognized as such and treated accordingly. The possibility of a cardiomyopathy should be considered in patients with cardiac disease who have had previous radiation to the mediastinum.

GLETON, R.T., WESLEY, M.N. \& WIERNIK, P.H. (1982). Longterm cardiovascular evaluation of patients with Hodgkin's disease treated by thoracic mantle radiation therapy. Cancer Treatment Reports, 66, 1003.

BROSIUS, F.C., WALLER, B.F. \& ROBERTS, W.C. (1981).

Radiation heart disease: analysis of 16 young (aged 15-33 
years) necropsy patients who received over 3,500 rads to the heart. American Journal of Medicine, 70, 519.

BURCH, G.E., SOHAL, R.S., SUN, S.C., MILLER, G.C. \& COLCOLOUGH, H.L. (1968). Effects of radiation on the human heart: an electron microscopic study. Archives of Internal Medicine, 121, 230.

BURNS, R.J., BAR-SCHLOMO, B.Z., DRUCK, M.N., HERMAN, J.G., GILBERT, B.W., PERRAULT, D.J. \& McLAUGHLIN, P.R. (1983). Detection of radiation cardiomyopathy by gated radionuclide angiography. American Journal of Medicine, 74, 297.

CARMEL, R.C. \& KAPLAN, H.S. (1976). Mantle irradiation in Hodgkin's disease. An analysis of technique, tumour eradication and complications. Cancer, 37, 2813.

COHN, K.E., STEWART, J.R., FAJARDO, L.F. \& HANCOCK, E.W. (1967). Heart disease following radiation. Medicine, 46, 281.

DOLLINGER, M.R., LAVINE, D.M. \& FOYE, L.V. (1966). Myocardial infarction due to post irradiation fibrosis of the coronary arteries. Care of successfully treated Hodgk- in's disease with lower esophageal involvement. Journal of the American Medical Association, 195, 176.

GOTTDIENER, J.S., KATIN, M.J., BOVER, J.S., BACHARACH, S.L. \& GREEN, M.V. (1983). Late cardiac effects of $\subseteq$ therapeutic mediastinal irradiation. Assessment by echocardiography and radionuclide angiography. New England Journal of Medicine, 308, 567.

MCREYNOLDS, R.A., GOLD, G.L. \& ROBERTS, W.C. (1976). Coronary heart disease after mediastinal irradiation for Hodgkin's disease. American Journal of Medicine, 60, 39.

RUBIN, E., CAMARA, J., GRAYZEL, D.M. \& ZAK, F.G. (1963). Radiation-induced cardiac fibrosis. American Journal of Medicine, 34, 71.

STEWART, J.R. \& FAJARDO, L.F. (1971). Radiation induced heart disease; clinical and experimental aspects. Radiology Clinics of North America, 9, 511.

TRAC.Y, G.P., BROWN, D.E., JOHNSON, L.W. \& GOTTLIEB, A.J. (1974). Radiation-induced coronary artery disease. Journal of the American Medical Association, 288, 1660. 